\title{
Automatic Conformal Anti-radial Ultrasound Scanning for Whole Breast Screening
}

\author{
Ching-Yen Lee ${ }^{1}$ Pai-Chi Li ${ }^{1,2}$ (1)
}

Received: 25 August 2018 / Accepted: 11 June 2019 / Published online: 1 July 2019

(c) The Author(s) 2019

\begin{abstract}
Background Anti-radial ultrasound scanning is one of the main scanning approaches used in ultrasound breast screening. It can be used for cross-sectional imaging of mammary ductal/lobular tissue and provide information about suspicious tissue. It has certain advantages compared to linear scanning, but automatic anti-radial scanning is not yet available. Our goal is to propose an ultrasound scanning system for whole breast anti-radial scanning.

Methods We previously developed an automatic ultrasound scanning system for whole breast screening involving linear scanning. The present study builds on our previous work by incorporating (1) surface-reconstruction algorithms, (2) a rotatable holder design, and (3) a scan-path-smoothing algorithm to achieve conformal anti-radial scanning.

Results An improvement of approximately $40 \%$ in the normal-vector estimation is obtained with our new method, and the scan stability is improved by the scan-path-smoothing algorithm. 3D volume data of each scan are available.

Conclusions We have successfully developed an automatic ultrasound scanning system for anti-radial breast scanning. This type of system, which has not been reported previously in the literature, can be an effective tool for fully automatic ultrasound breast screening.
\end{abstract}

Keywords Anti-radial Scan $\cdot$ Surface reconstruction $\cdot$ Scan-path smoothing $\cdot$ Ultrasound conformal scanning

\section{Introduction}

Ultrasound is an important imaging modality for breast screening due to several advantages. For example, it is noninvasive, free of ionizing radiation, and can be performed in real time. In addition, it has good contrast compared to mammograms in dense breast [1-6]. Clinically, three hand-held scanning approaches are commonly used: linear (also called raster), radial, and anti-radial scanning [7]. The linear scan is the most common way to obtain the sagittal and transverse planes of the breast. Nonetheless, the cross-sectional plane of the mammary duct, which is clinically important, is also

Pai-Chi Li

paichi@ntu.edu.tw

Ching-Yen Lee

gp6fu6@gmail.com

1 Graduate Institute of Biomedical Electronics and Bioinformatics, National Taiwan University, Taipei, Taiwan

2 Department of Electrical Engineering, National Taiwan University, Taipei, Taiwan important and should be obtained by using anti-radial scanning [7-10].

Since whole breast ultrasound screening is performed manually by radiologists and sonographers, the obtained results are highly dependent on the experience of the operators. To reduce the influence of human factors in ultrasound examinations, robot-assisted systems have been designed [11-14]. Automated breast ultrasound screening (ABUS) and automated whole breast ultrasound (AWBU) [15] are robotic medical systems that are specified designed for breast screening. Based on the system designs, breast ultrasound can be performed either semiautomatically or guided by a moving structure. On contrast to ABUS and AWBU, the $\mathrm{iVu}$ system [16] was developed for breast screening using radial scanning. Despite the importance of fitting the body shape, none of the existing systems focus on an adaptive scan path: a predetermined curved scan pattern is chosen by ABUS and $\mathrm{iVu}$, and the orientation of the transducer in AWBU is handled and guided by the operator. The functionality of the available commercialized systems for breast screening is limited by these predetermined scan trajectories. 


\subsection{Goals of This Study}

To the best of our knowledge, only linear and radial scanning products are available commercially. Here we propose an ultrasound scanning system to achieve anti-radial scanning for whole breast screening. Previously we have developed a conformal ultrasound scanning system for linear scanning only. The system setup is shown in Fig. 1, and details about the operation of the developed system are available elsewhere [17]. In brief, the system enables the generation of a scanning path for each individual examinee based on the spatial information sensed by a 3D camera, and a robotic arm with six degrees of freedom is used to perform conformal whole breast scanning. A customized transducer holder was designed for connecting the robotic arm and the ultrasound transducer of a portable ultrasound scanning system. The present study focused on achieving anti-radial breast scanning by (1) modifying the existing algorithms and (2) improving the design of the transducer holder. In addition, we generated the corresponding 3D volume data for each individual scan.

\section{Materials and Methods}

A newly designed transducer holder was introduced, and the surface reconstruction and scan-path-smoothing algorithms were proposed for better approximating the target surface and to improve the scan stability, respectively. The new system allows the 3D volume of the scanned ultrasound images to be generated. The system performance was evaluated both using simulations and by scanning a breast phantom.

\subsection{Rotatable Transducer Holder for Anti-radial Scanning}

The transducer holder designed in the previous study could only perform linear scanning, and using the existing setup for other types of scan would damage the instruments, such as bending the wires of the transducer. Here we propose a modified rotatable transducer holder design consisting of a stepping motor (Faulhaber, Schönaich, Germany), aluminum supports, a rotating structure, and connector made by a 3D printer, as shown in Fig. 2. The operating orientation of the transducer is maintained by the rotating structure and the stepping motor. The resolution of the stepping motor is $1.8^{\circ}$, which is sufficient to match the system's design criteria. Movement of the rotating structure is handled by a bearing (THK, Tokyo, Japan) with an inner radius of $80 \mathrm{~mm}$. Cooperation between the stepping motor and rotating structure allows the scanning transducer to rotate $\pm 90^{\circ}$.

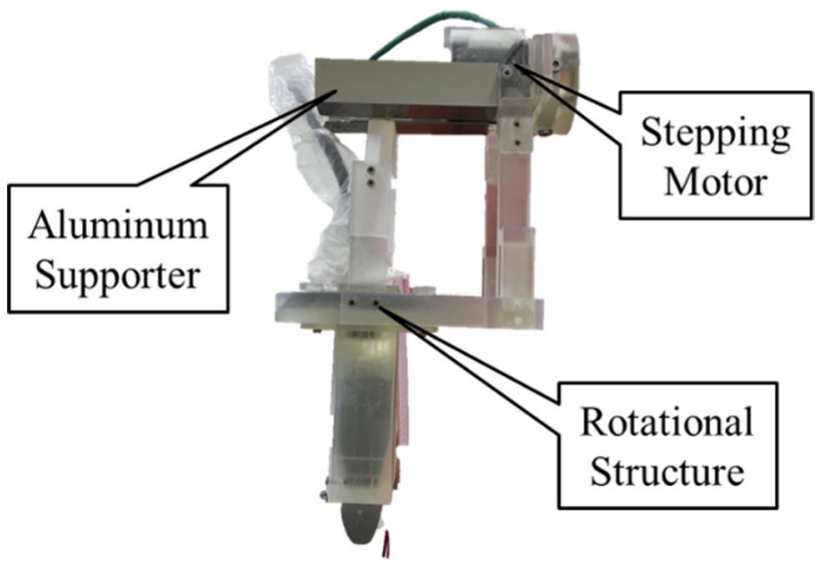

Fig. 2 The modified transducer holder for anti-radial scanning
Fig. 1 The developed automatic conformal ultrasound scanning system for whole breast screening

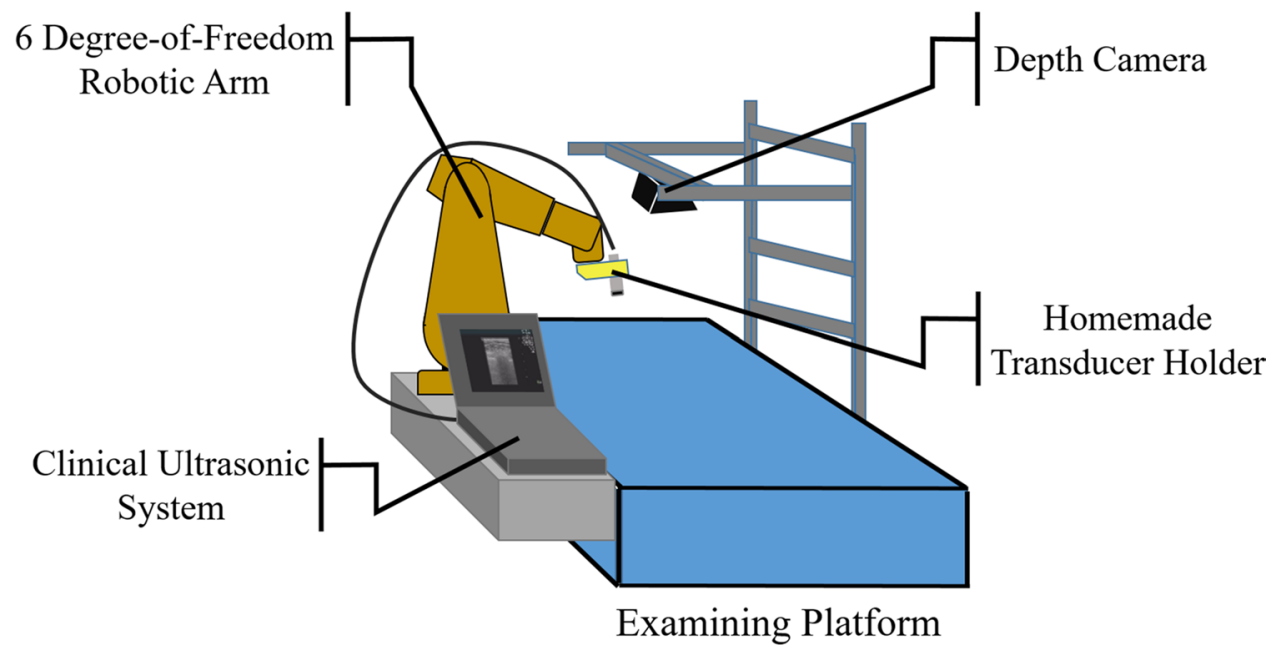




\subsection{Breast Phantom}

A breast phantom (CIRS Model 052A, Norfolk, VA, US) designed to mimic the shape of the breast and the properties of amorphous lesions was used to evaluate the performance of the proposed system both using simulations and by scanning a breast phantom.

\subsection{Algorithms}

In our previous work we employed temporal median/mean filters to reduce the noise level of the $3 \mathrm{D}$ camera. The corresponding normal vector (estimated by principalcomponents analysis) and linear scan path (based on the desired conditions) were generated based on the detected surface. However, the effects of noise could not be effectively reduced, and the estimated normal vector exhibited a standard deviation of $\sim 1.8^{\circ}$ to $6.4^{\circ}$, which resulted in unnecessary movement of the robotic arm during scanning.

Algorithm development in the present study focused on (1) improving the scanning stability by using a better surface smoothing/approximation method, (2) generating the anti-radial scan path adapted to the target surface, and (3) visualizing the scan result with 3D volume data.

\section{Spatial Data Smoothing for Target Surface Reconstruction}

This study employed a combination of two smoothing methods (a bilateral filter and 2D B-spline) for mitigating the spatial error of the 3D camera [18]. Generally, a bilateral filter is a modified Gaussian filter that uses the difference in distance and pixel intensity as weights. In our approach, the pixel intensity represents the distance measured by the 3D camera, and the bilateral filter can be expressed by

$I_{s}(x, y)=\frac{\sum_{i=-w / 2}^{w / 2} \sum_{j=-w / 2}^{w / 2} G_{s}(i, j) \cdot G_{i}(i, j) \cdot I(x+i, y+j)}{\sum_{i=-w / 2}^{w / 2} \sum_{j=-w / 2}^{w / 2} G_{s}(i, j) \cdot G_{i}(i, j)}$

$G_{s}(i, j)=e^{-\frac{i^{2}+j^{2}}{2 \sigma_{s}^{2}}}$

$G_{i}(i, j)=e^{-\frac{((x, y)-I(x+i, y+j))^{2}}{2 \sigma_{i}^{2}}}$

where $w$ is the window size, $I$ and $I_{s}$ represent the distances of the measured and processed values, respectively, and $G_{s}$ and $G_{i}$ are Gaussian weightings, which can be calculated by the Gaussian distribution with standard deviations $\sigma_{s}$ and $\sigma_{i}$, respectively. A B-spline is a curve-smoothing algorithm that can be modeled by the Knot vector $(V)$, controls $(P)$, and $k$ th-order basis functions. The Knot vector is a sequential set of values from 0 to the number of outputs:

$V=\left\{0, \cdots, v, \cdots, v_{\max }\right\}$.

The $k$ th order basis function, $N_{i, k}$, can be defined recursively:

$N_{i, k}(v)=\frac{v-v_{i}}{v_{i+k-1}-v_{i}} N_{i, k-1}(v)+\frac{v_{i+k}-v}{v_{i+k}-v_{i+1}} N_{i+1, k-1}(v)$

$N_{i, 0}(v)=\left\{\begin{array}{rr}1, & \text { if } v_{i}<v<v_{i+1} \\ 0, & \text { otherwise }\end{array}\right.$.

The B-spline curve and $k$ th-order basis function can be defined as follows:

$P(v)=\sum_{i=0}^{n} P_{i} N_{i, k}(v), 0<v<v_{\max }$

The 2D B-spline (B-spline surface) can be defined with parameters $(u, v)$ with $n+1$ and $m+1$ control points as follows:

$P(u, v)=\sum_{j=0}^{m} \sum_{i=0}^{n} P_{i, j} N_{i, k}(v) M_{j, k}(u)$

In this study we used a bilateral filter to address the noise issue, and a 2D B-spline was used to estimate the pixel values that could not be precisely detected.

\section{Principal Component Analysis for Normal-Vector Estimation}

The ultrasound transducer should remain perpendicular to the target surface during automatic scanning. This study used principle-components analysis to estimate the normal vectors of the surface. With the target coordinate $\left(P_{\text {target }}=\left[x_{\text {target }}, y_{\text {target }}, z_{\text {target }}\right]\right)$ on the surface, the corresponding normal vector can be derived based on the surrounding coordinates $\left(P_{i}=\left[x_{i}, y_{i}, z_{i}\right]\right)$. The $n \times 3$ matrix, PCA, can be obtained by subtracting $P_{\text {target }}$ from $P_{i}$ - as shown below:

PCA $=\left[\begin{array}{c}P_{1}-P_{\text {target }} \\ P_{2}-P_{\text {target }} \\ \vdots \\ P_{n}-P_{\text {target }}\end{array}\right]=\left[\begin{array}{c}x_{1}-x_{\text {target }} y_{1}-y_{\text {target }} z_{1}-z_{\text {target }} \\ x_{2}-x_{\text {target }} y_{2}-y_{\text {target }} z_{2}-z_{\text {target }} \\ \vdots \\ x_{n}-x_{\text {target }} y_{n}-y_{\text {target }} z_{n}-z_{\text {target }}\end{array}\right]$

Then, square matrix $\mathrm{M}$ can be derived as 
$\mathrm{M}=(\mathrm{PCA})^{\mathrm{T}} \cdot \mathrm{PCA}$.

After computing the eigenvalues and eigenvectors of $\mathrm{M}$, the first two eigenvectors can be used to span the target surface, and the corresponding normal vector can be generated by the cross product of the two eigenvectors. Figure 3 illustrates the relationships between the target coordinate, the surrounding coordinates, and the eigenvalues and eigenvectors.

\section{Generation of the Anti-radial Scan Path}

The desired scanning region was selected manually before processing, and then the center of the selected scanning region could be obtained. The anti-radial scan begins in the horizontal direction. The orientation of each following scan is increased by an angle offset, $\theta$. The contact angles, based on the reconstructed surface, were obtained by principle-components analysis. The development of the anti-radial scan path is shown in Fig. 4. In an anti-radial scan, moving across the cross-center can minimize the total scan time and the overall required movement.

\section{Model-Based Scan-Path Smoothing}

To further improve the smoothness of the variance of the normal vectors on the scan path, we propose evaluating the normal vectors using the component ratio defined as

ratio $_{\mathrm{i}}=\left.\frac{\mathrm{nv}_{\mathrm{i}}}{\operatorname{norm}(\mathrm{nv})}\right|_{i=x, y, z}$
Fig. 3 Illustration of principlecomponents analysis for normal-vector estimation

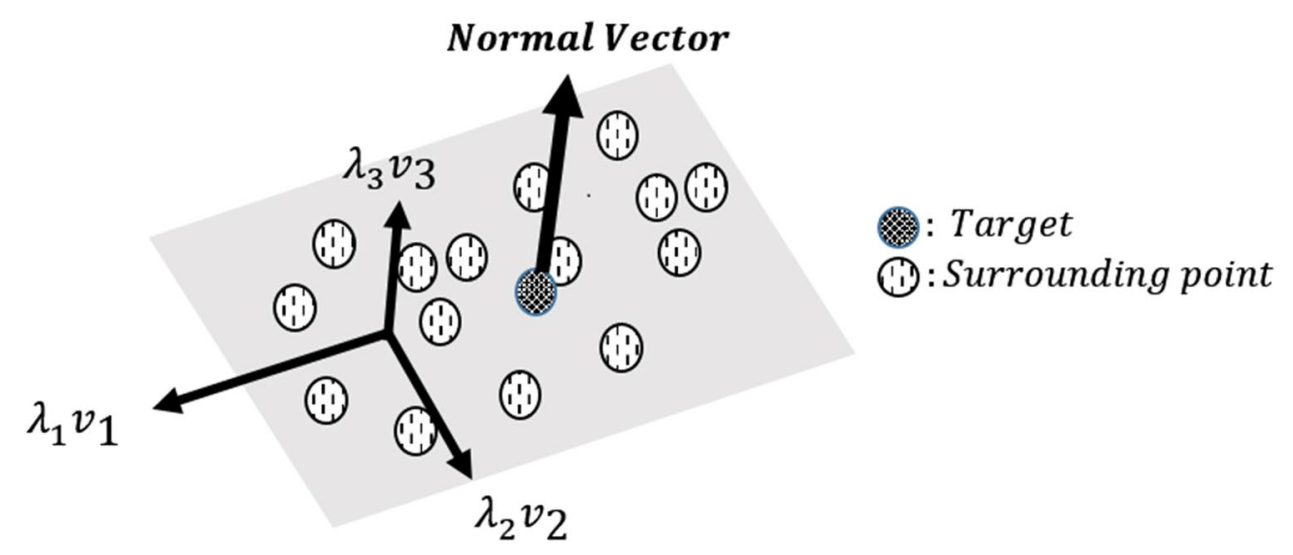

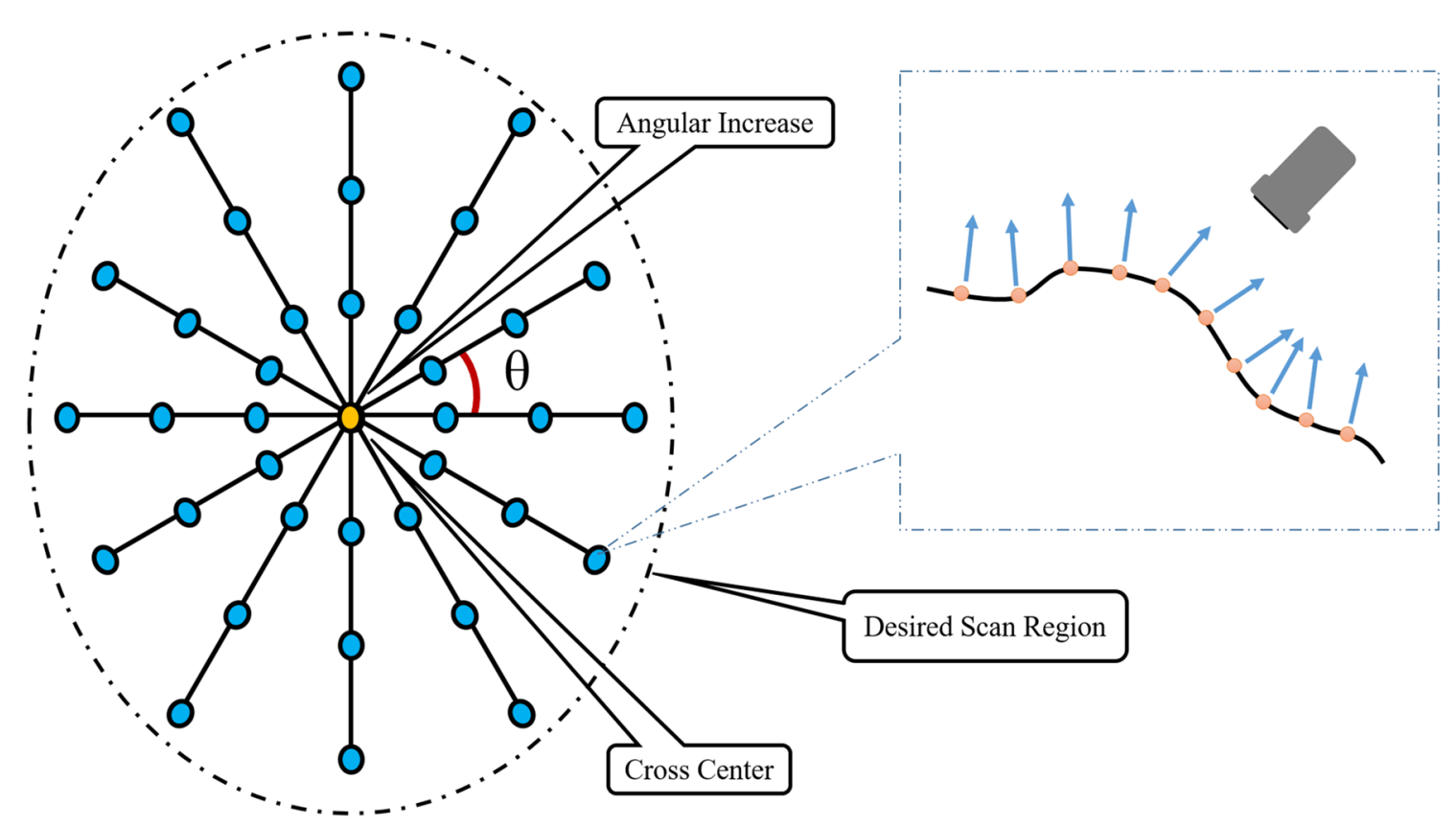

Fig. 4 The scan pattern for generating an anti-radial scan 
where nv denotes the normal vector $\left[v_{x}, v_{y}, v_{z}\right], \mathrm{nv}_{i}$ represents the $x, y$, and $z$ components of $\mathrm{nv}$, and the norm operation is $\sqrt{v_{x}^{2}+v_{x}^{2}+v_{x}^{2}}$. A spherical surface was used to simulate the breast geometry. In addition, a straight path and the corresponding normal vectors represent the scan trajectory and the contact angle (Fig. 5a). The $x, y$, and $z$ component ratios on the straight line are plotted in Fig. 5b, which indicates that both the $x$ and $y$ component ratios can be approximated by linear equations, and the $z$ component can be approximated by a quadratic equation.

We used the least-squares error (LSE) to approximate both the linear and quadratic equations. As shown in Fig. 5b, the ratio components of $x$ and $y$ can be approximated by linear equations:

$y=m x+c$

The error of each measured coordinate and error summation can be denoted as

$\left.E=\sum_{i}\left(y_{i}-y\right)^{2}=\sum_{i}\left(y_{i}-m x-c\right)\right)^{2}$

To minimize the total error, partial differentiation is performed with respect to $m$ and $c$ :

$\frac{\partial E}{\partial m}=0 \Rightarrow m \sum_{i} x_{i}^{2}+c \sum_{i} x_{i}=\sum_{i} x_{i} y_{i}$

$\frac{\partial E}{\partial c}=0 \Rightarrow m \sum_{i} x_{i}+c n=\sum_{i} y_{i}$

and $m$ and $c$ can be solved through a matrix operation:

$\mathrm{A} \cdot\left[\begin{array}{c}\mathrm{m} \\ \mathrm{c}\end{array}\right]=\mathrm{B} \Rightarrow\left[\begin{array}{c}\mathrm{m} \\ \mathrm{c}\end{array}\right]=\mathrm{B} / \mathrm{A}$

where the components of $\mathrm{A}$ and $\mathrm{B}$ can be obtained by
$\mathrm{A}=\left[\begin{array}{cc}\sum_{i} x_{i}^{2} & \sum_{i} x_{i} \\ \sum_{i} x_{i} & n\end{array}\right]$

$\mathrm{B}=\left[\begin{array}{c}\sum_{i} x_{i} y_{i} \\ \sum_{i} y_{i}\end{array}\right]$.

Then the values of $m$ and $c$ in Eq. 12 can be obtained using the following equation; in other words, the two curves ( $x$ and $y$ ) in Fig. 5 b can be approximated:

$\left[\begin{array}{c}\mathrm{m} \\ \mathrm{c}\end{array}\right]=\mathrm{A}^{-1} \cdot \mathrm{B}$

As shown in Fig. 5b, the ratio component of $z$ can be approximated by a quadratic equation:

$y=a x^{2}+b x+c$.

The error of each measured coordinate and error summation can be denoted as follows:

$\left.E=\sum_{i}\left(y_{i}-y\right)^{2}=\sum_{i}\left(y_{i}-a x^{2}-b x-c\right)\right)^{2}$.

To minimize the total error, partial differentiation is performed with respect to $a, b$, and $c$ :

$\frac{\partial E}{\partial a}=0 \Rightarrow a \sum_{i} x_{i}^{4}+b \sum_{i} x_{i}^{3}+c \sum_{i} x_{i}^{2}=\sum_{i} x_{i}^{2} y_{i}^{2}$

$\frac{\partial E}{\partial b}=0 \Rightarrow a \sum_{i} x_{i}^{3}+b \sum_{i} x_{i}^{2}+c \sum_{i} x_{i}=\sum_{i} x_{i} y_{i}^{2}$

$\frac{\partial E}{\partial c}=0 \Rightarrow a \sum_{i} x_{i}^{2}+b \sum_{i} x_{i}+c n=\sum_{i} y_{i}^{2}$

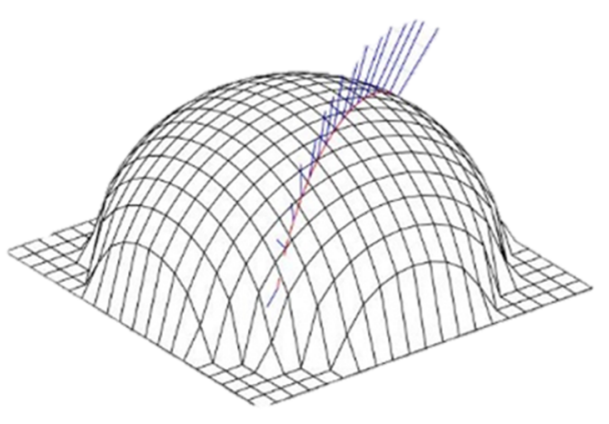

(a)
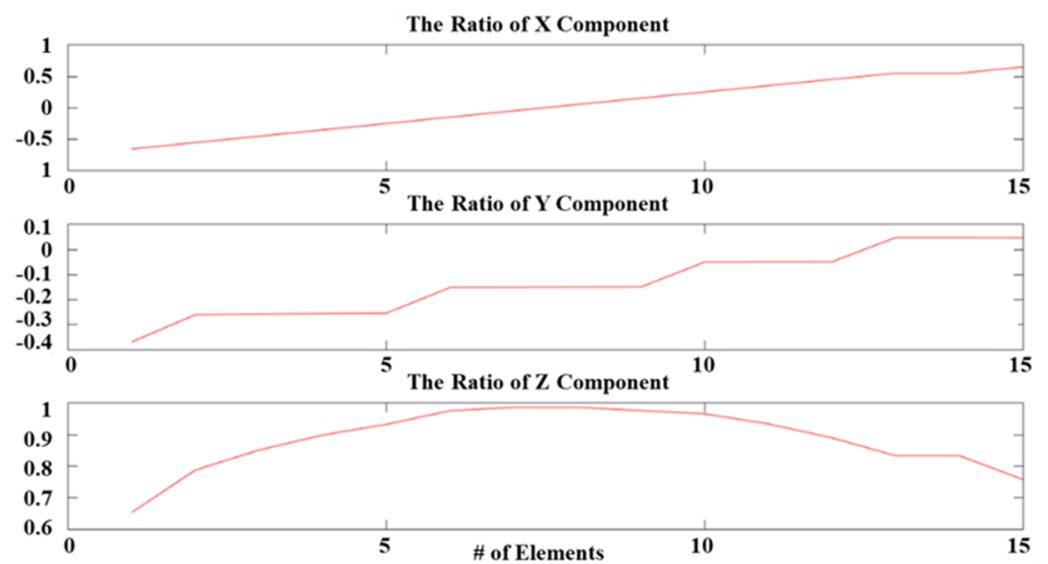

(b)

Fig. 5 a The ideal spherical surface and the corresponding normal vector. b Ratios of the $x, y$, and $z$ components 
and $a, b$, and $c$ can be solved using a matrix operation:

$\mathrm{A} \cdot\left[\begin{array}{l}a \\ b \\ c\end{array}\right]=\mathrm{B} \Rightarrow\left[\begin{array}{l}a \\ b \\ c\end{array}\right]=\mathrm{B} / \mathrm{A}$

where the components of A and B can be obtained by

$$
\begin{aligned}
\mathrm{A} & =\left[\begin{array}{ccc}
\sum_{i} x_{i}^{4} & \sum_{i} x_{i}^{3} & \sum_{i} x_{i}^{2} \\
\sum_{i} x_{i}^{3} & \sum_{i} x_{i}^{2} & \sum_{i} x_{i} \\
\sum_{i} x_{i}^{2} & \sum_{i} x_{i} & n
\end{array}\right] \\
\mathrm{B} & =\left[\begin{array}{c}
\sum_{i} x_{i}^{2} y_{i}^{2} \\
\sum_{i} x_{i} y_{i}^{2} \\
\sum_{i} y_{i}^{2}
\end{array}\right] .
\end{aligned}
$$

Then the values of $a, b$, and $c$ in Eq. 20 can be obtained using the following equation; in other words, the $z$ curve in Fig. 5 b can be approximated:

$$
\left[\begin{array}{l}
a \\
b \\
c
\end{array}\right]=\mathrm{A}^{-1} \cdot \mathrm{B}
$$

With these approximations, the normal vector described in Eq. 11 can be expressed as follows:

$n v_{i}=\frac{\left[\begin{array}{l}\operatorname{ratio}_{\mathrm{x}, \mathrm{i}} \\ \operatorname{ratio}_{\mathrm{y}, \mathrm{i}} \\ \operatorname{ratio}_{\mathrm{z}, \mathrm{i}}\end{array}\right]}{\sqrt{\text { ratio }_{\mathrm{x}, \mathrm{i}}^{2}+\text { ratio }_{\mathrm{y}, \mathrm{i}}^{2}+\text { ratio }_{\mathrm{z}, \mathrm{i}}^{2}}}$.

\section{3D Volume Data Reconstruction}

The spatial information of each scanned ultrasound image is recorded during the conformal scan, which can be used to align the scan results. A mesh-grid volume data set is created for the reconstruction of volume data. Each pixel of the ultrasound image can be mapped onto the data expression grid, as shown in Fig. 6.

The coordinate expression of each pixel can be represented as

$\mathbf{P}_{1}=\left[\begin{array}{c}x_{i} \\ y_{i} \\ z_{i}\end{array}\right]$

The intensity of each pixel is determined based on its eight surrounding nodes, and the intensity of each node can be obtained using the distance weighting sum:

Volume_intensity $(\mathrm{x}, \mathrm{y}, z)=\frac{\sum_{i} \mathrm{dist}_{\mathrm{i}} \cdot \text { intensity }_{\mathrm{i}}}{\sum_{i} \text { dist }_{\mathrm{i}}}$

Dist $=$ norm $\left(\right.$ target $\left.-\mathrm{P}_{\mathrm{i}}\right)$.

\section{Performance Evaluation Results}

\subsection{Surface Reconstruction}

Two ideal surfaces (flat and spherical) with added random noise were used to simulate the surfaces captured by the 3D camera. The bilateral filter was modeled by $w=3, \sigma_{s}=2$, and $\sigma_{i}=1$ (as defined in Eqs. 1 to 3). The 2D B-spline had

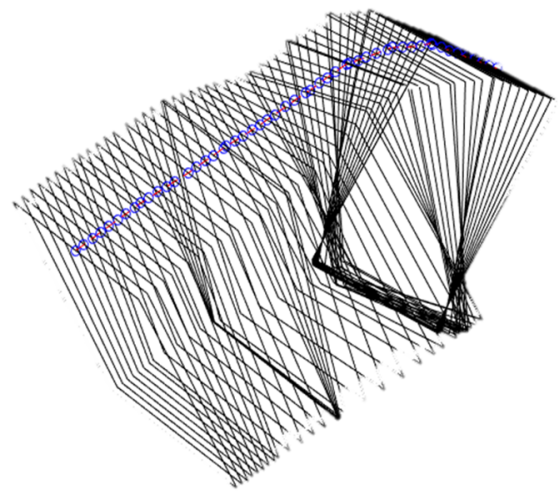

(a)

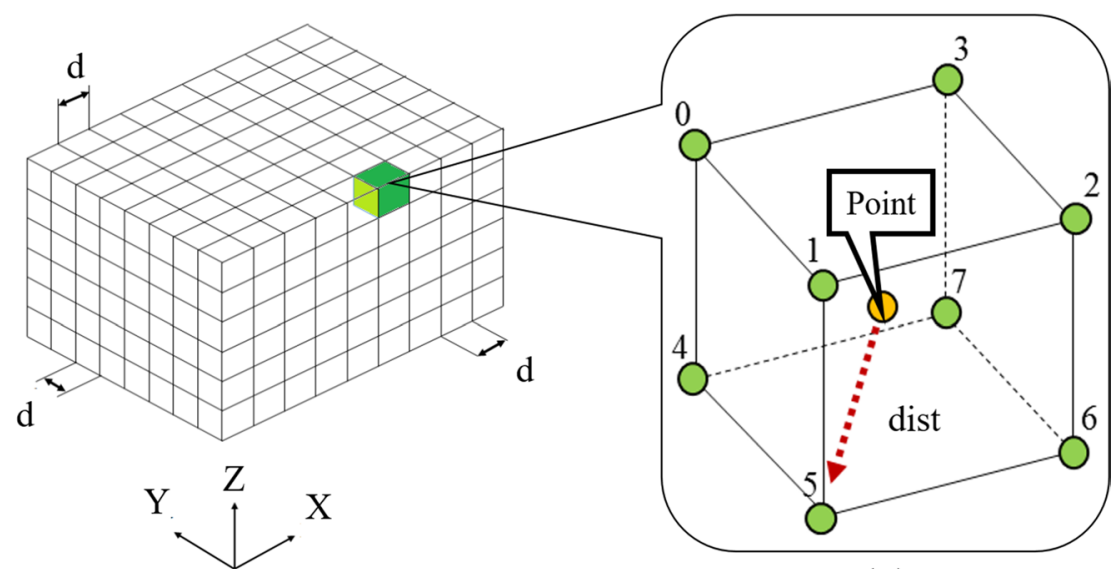

(b)

(c)

Fig. 6 a 3D alignment of scan results. b Volume grid for 3D reconstruction. c Definition of the distance of a pixel to each corner 
Table 1 MSE/standard-deviation values for different filter configurations

\begin{tabular}{lll}
\hline Surface & Flat surface $(\mathrm{mm})$ & $\begin{array}{l}\text { Spherical } \\
\text { surface } \\
(\mathrm{mm})\end{array}$ \\
\hline Noise & $0.47 / 0.29$ & $0.50 / 0.28$ \\
Bilateral filter & $0.47 / 0.08$ & $0.49 / 0.16$ \\
2D B-spline & $0.47 / 0.15$ & $0.48 / 0.18$ \\
Bilateral filter+2D & $0.47 / 0.06$ & $0.48 / 0.14$ \\
B-spline & & \\
\hline
\end{tabular}

an order of 3. The processed results were compared with the ideal surfaces. The mean squared error (MSE) and standard deviation were used to evaluate the coordinate differences between the reference and filtered surfaces. There was little improvement in the MSE (up to 4\%), but the standard deviations of both surfaces were reduced substanti-ally (50-80\%), and so fewer adjustments were needed to the position of the transducer while scanning. The comparisons are presented in more detail in Table 1 and Fig. 7.

\subsection{Improvement in Normal-Vector Estimation}

The normal vector was estimated using principle-components analysis and the cross product with the same surface conditions as mentioned above. The error in normal-vector estimation is defined by the angle difference between the reference and estimated normal vectors. Using principlecomponents analysis resulted in a higher accuracy and lower standard deviation than when using the cross product, with typically 35-40\% improvement being achieved by processing with principle-components analysis. More details are presented in Table 2 and Fig. 7.

\subsection{Model-Based Scan-Path-Smoothing Algorithm}

The spatial-data-smoothing algorithm can successfully reduce the noise level of the reconstructed surface and the corresponding normal vector (as mentioned in Sect. 8.1), but the proposed model-based scan-path-smoothing algorithm aimed to further reduce unnecessary vibration caused associated with the standard deviation or angle error. The improvement obtained by using this algorithm was verified using both the simulated surface and actual data from the breast phantom. Figure $8 \mathrm{a}$ and $\mathrm{c}$ show the original normal vectors, while Fig. $8 b$ and d display the smoothed normal vectors; these visualizations demonstrate that the estimated normal vector can be successfully smoothed.

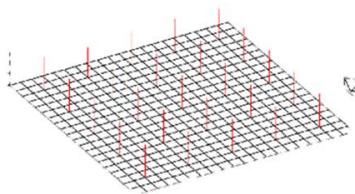

(a)

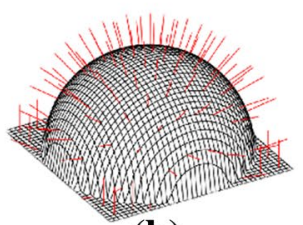

(b)

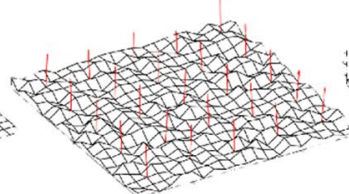

(c)

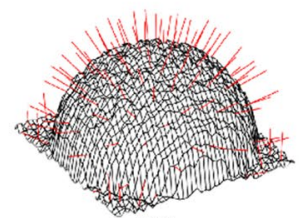

(d)

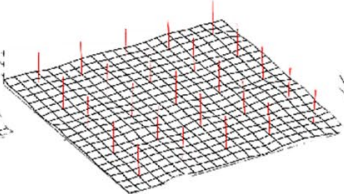

(e)

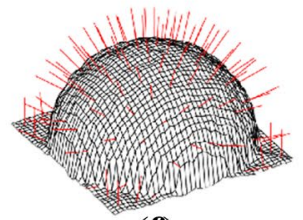

(f)

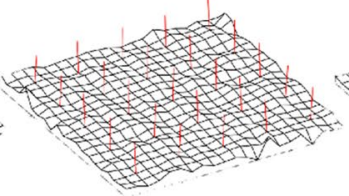

(g)

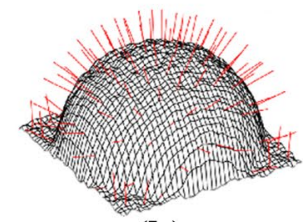

(h)

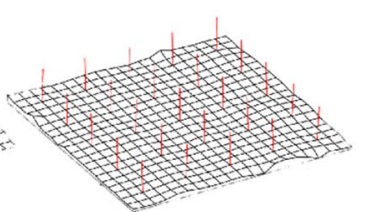

(i)

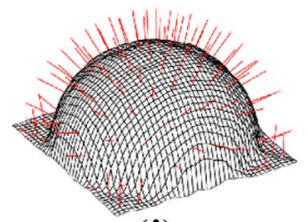

(j)
Fig. 7 Visualization of normal-vector estimation by principle-components analysis. a, b Reference surfaces. c, d Simulated surfaces detected by the $3 \mathrm{D}$ camera. Reconstructed surfaces after smoothing using a bilateral filter (e, f), a 2D B-spline (g, h), and a bilateral filter plus a 2D B-spline $(\mathbf{i}, \mathbf{j})$
Table 2 MSE/standarddeviation values for the estimations of normal vectors

\begin{tabular}{llllll}
\hline Surface & Method & \multicolumn{2}{l}{ Estimated error, degrees } & \\
\cline { 3 - 6 } & & Noise & Bilateral & 2D B-spline & $\begin{array}{l}\text { Bilateral } \\
\text { filter + 2D } \\
\text { B-spline }\end{array}$ \\
\hline Flat & Principle-components analysis & $2.83 / 1.39$ & $1.73 / 0.86$ & $2.42 / 1.36$ & $1.64 / 0.90$ \\
Spherical & Principle-components analysis & $2.62 / 2.51$ & $1.47 / 1.48$ & $1.94 / 1.77$ & $1.58 / 1.51$ \\
\hline
\end{tabular}




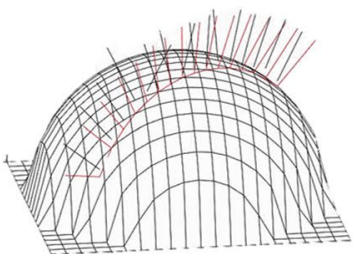

(a)

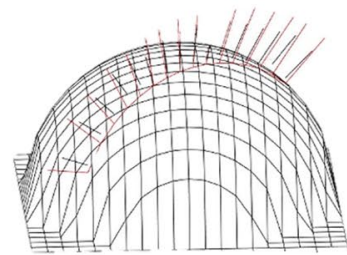

(b)

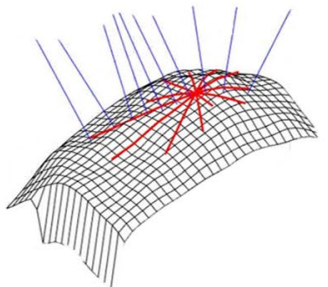

(c)

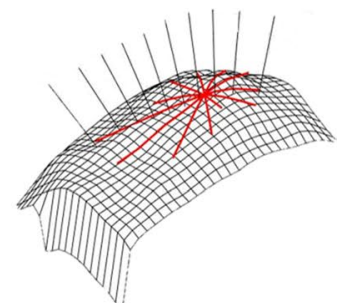

(d)

Fig. 8 a, c Normal vectors with respect to the simulated and actual surfaces. b, d Results obtained by smoothing using the model-based scanpath-smoothing algorithm

\subsection{Anti-radial Scanning and 3D Volume Data Reconstruction}

Anti-radial scanning with 30 angle increments and a total of 6 scan paths was performed on the breast phantom. The scanning stability was improved when scanning a circular region with a radius of $50 \mathrm{~mm}$ at a scan speed of $5 \mathrm{~mm} / \mathrm{s}$. A total scan time of around $3.5 \mathrm{~min}$ was required in the current system setup. 3D volume data were generated based on the recorded spatial data. Figure 9a shows one of the six reconstructed 3D data volumes. To further verify the reconstructed results, one section of each reconstruction was chosen and compared with the actual ultrasound image of the breast phantom in Fig. 9b-m.

\section{Discussion}

We have successfully performed anti-radial scanning, improved the scan stability, and visualized the scan results in 3D volume representations using our modified system. However, there are some limitations that cannot be overcome using with the current prototype.

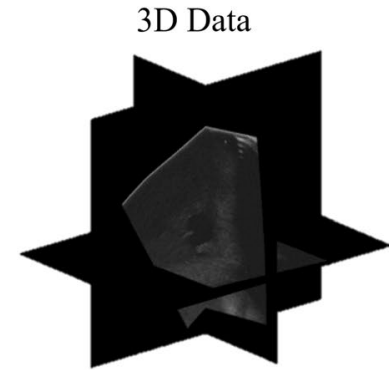

(a)

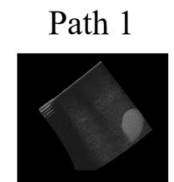

(b)

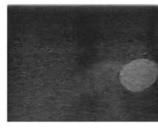

(h)

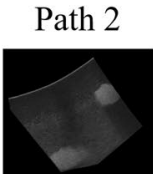

(c)

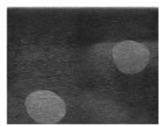

(i)

\subsection{Limitations of the Current System Setup for Anti-radial Scanning}

An additional rotation, as allowed by the modified transducer holder, minimized the requirement for a posture change in the anti-radial scan. Nonetheless, additional $100-\mathrm{mm}$ and 250-mm extensions along the $x$ - and $z$-axes were unavoidable because of the complexity of the holder design. Also, the effective scan region is restricted to within a circular region with a radius of less than $50 \mathrm{~mm}$.

Choosing the appropriate scanning speed is important to obtain the optimal trade-off between the spatial resolution and the posture changes of the robot arm. By scanning at $5 \mathrm{~mm} / \mathrm{s}$, a spatial resolution of $0.67 \mathrm{~mm}$ can be obtained, but results in a processing time of around $3.5 \mathrm{~min}$. Increasing the scan speed will reduce the scan accuracy (spatial resolution) and scan stability (due to larger changes in the posture of the robot arm). For processing at a higher scan speed, an ultrasound system with a higher frame acquisition rate (e.g., $100 \mathrm{fps}$ ) is suggested. On the other hand, processing with a smaller transducer holder can reduce the required posture changes of the robot arm while scanning. In addition, the algorithm for determining the scan region in anti-radial scanning was not addressed in this study due to the large variation between examinees. To avoid any improper determinations, manually selecting the scan region is suggested.

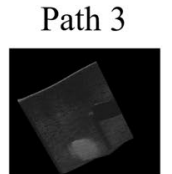

(d)

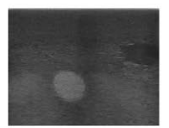

(j)

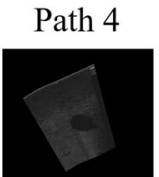

(e)

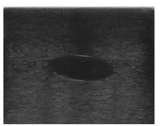

(k)

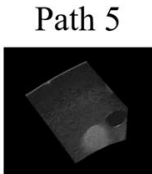

(f)

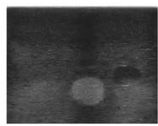

(l)

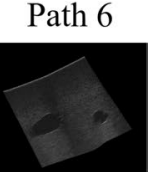

(g)

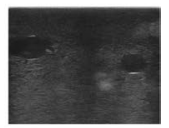

(m)

Fig. 9 a A reconstructed 3D data volume. Comparisons between the cross sections of the 3D reconstruction data (b-g) and the actual ultrasound images $(\mathbf{h}-\mathbf{m})$ 


\subsection{Performance of the Proposed Algorithms}

The accuracy of our proposed method was tested using ideal flat and spherical surfaces. The results show that the proposed algorithm can reduce the standard deviation of the estimated error and improve the smoothness of the estimated normal vector. The proposed filter configuration (bilateral filter $+2 \mathrm{D}$ B-spline) reduced the estimated errors by $50 \%$, which is the key point for smoothing the reconstructed surface. Based on the smoothed reconstructed surface, the principle-components analysis method can provide the estimated normal vector with a lower angle standard deviation (reduced from $2.51^{\circ}$ to $1.51^{\circ}$, as indicated in Table 2). The reconstruction results also show that the proposed system can smoothly move across the breast phantom surface without unnecessary disturbances.

\subsection{Deformation of the Breast Phantom}

Due to deformation of the breast phantom, differences in the geometry between the reconstructed surface and the actual images are unavoidable. This deformation issue was alleviated in our study in two ways. First, our general setup includes a contact force sensor to make sure that the ultrasound transducer establishes good contact with the skin while avoiding applying an excessive force as often seen during manual scanning. In other words, our use of a force sensor minimized the deformation. Second, the image object (i.e., breast) can be easily confined and fixed during the scanning by using a large membrane covering the entire breast. This is already used in ABUS by applying a large transducer holder and a scanning frame on the breast. A similar scanning frame can also be used in our setup to avoid the deformation problem. The data presented in Tables 1 and 2 indicate the good accuracy (submillimeter) that can be achieved with our proposed approach.

\section{Conclusions}

This study developed a prototype of an automatic breast ultrasound screening system for anti-radial scanning. Such an automatic system is clinically important, and this is the first report of such a system in the literature. A modified transducer holder consisting of a stepping motor and rotating structure was designed to adjust the orientation of the transducer. The surface-model spatial-data-smoothing algorithm provided a better approximation of the target surface. The standard deviation of the surface reconstruction was effectively reduced (by 50-80\%) in the simulations of flat and spherical surfaces, while an improvement of $35-40 \%$ in angle accuracy was also achieved. Our model-based scanpath-smoothing algorithm further optimized the suggested contact angle based on the shape of the breast, and this was verified in breast phantom scans. Six-direction anti-radial scanning was performed within a circular region (with a radius of $50 \mathrm{~mm}$ ) of the breast phantom, and the overall processing time was approximately $3.5 \mathrm{~min}$. The $3 \mathrm{D}$ volume reconstruction of each scan path was obtained, and the quality of the reconstruction was verified in comparisons of actual ultrasound images obtained from the breast phantom.

Funding This study was funded by Ministry of Science and Technology, Taiwan (MOST 103-2221-E-002-016-MY3).

\section{Compliance with Ethical Standards}

Conflict of interest The authors declare that they have no conflict of interest.

Ethical Approval All procedures performed in the human scanning study were in accordance with the ethical standards of the institution.

Informed Consent Informed consent was obtained from each participant included in the study.

Open Access This article is distributed under the terms of the Creative Commons Attribution 4.0 International License (http://creativecommons.org/licenses/by/4.0/), which permits unrestricted use, distribution, and reproduction in any medium, provided you give appropriate credit to the original author(s) and the source, provide a link to the Creative Commons license, and indicate if changes were made.

\section{References}

1. Kolb, T. M., Lichy, J., \& Newhouse, J. H. (2002). Comparison of the performance of screening mammography, physical examination, and breast US and evaluation of factors that influence them: An analysis of 27,825 patient evaluations. Radiology, 225(1), 165-175. https://doi.org/10.1148/radiol.2251011667.

2. Benson, S. R. C., Blue, J., Judd, K., \& Harman, J. E. (2004). Ultrasound is now better than mammography for the detection of invasive breast cancer. The American Journal of Surgery, 188(4), 381-385. https://doi.org/10.1016/j.amjsurg.2004.06.032.

3. Kaplan, S. S. (2001). Clinical utility of bilateral whole-breast US in the evaluation of women with dense breast tissue. Radiology, 221(3), 641-649. https://doi.org/10.1148/radiol.2213010364.

4. Kolb, T. M., Lichy, J., \& Newhouse, J. H. (1998). Occult cancer in women with dense breasts: Detection with screening USdiagnostic yield and tumor characteristics. Radiology, 207(1), 191-199. https://doi.org/10.1148/radiology.207.1.9530316.

5. Leconte, I., Feger, C., Galant, C., Berlière, M., Berg, B. V., D'Hoore, W., et al. (2003). Mammography and subsequent wholebreast sonography of nonpalpable breast cancers: The importance of radiologic breast density. American Journal of Roentgenology, 180(6), 1675-1679. https://doi.org/10.2214/ajr.180.6.1801675.

6. Okello, J., Kisembo, H., Bugeza, S., \& Galukande, M. (2014). Breast cancer detection using sonography in women with mammographically dense breasts. BMC Medical Imaging, 14, 41. https ://doi.org/10.1186/s12880-014-0041-0.

7. Stavros, A. T., Rapp, C. L., \& Parker, S. H. (2004). Breast ultrasound. Philadelphia: Lippincott Williams \& Wilkins. 
8. Dixon, A. M. (2008). Breast ultrasound HOW, WHY and WHEN (1st ed.). Amsterdam: Elsevier.

9. Henningsen, C., Kuntz, K., \& Youngs, D. (2013). Clinical guide to sonography (2nd ed.). Amsterdam: Elsevier.

10. Madjar, H., Rickard, M., Jellins, J., \& Otto, R. (1999). IBUS guidelines for the ultrasonic examination of the breast. European Journal of Ultrasound, 9(1), 99-102. https://doi.org/10.1016/ S0929-8266(99)00016-6.

11. Abolmaesumi, P., Salcudean, S. E., Zhu, W.-H., Sirouspour, M. R., \& DiMaio, S. P. (2002). Image-guided control of a robot for medical ultrasound. IEEE Transactions on Robotics and Automation, 18(1), 11-23. https://doi.org/10.1109/70.988970.

12. Janvier, M. A., Durand, L.-G., Cardinal, M.-H. R., Renaud, I., Chayer, B., Bigras, P., et al. (2008). Performance evaluation of a medical robotic 3D-ultrasound imaging system. Medical Image Analysis, 12(3), 275-290. https://doi.org/10.1016/j.media 2007.10.006.

13. Pierrot, F., Dombre, E., Dégoulange, E., Urbainb, L., Caron, P., Boudet, S., et al. (1999). Hippocrate: A safe robot arm for medical applications with force feedback. Medical Image Analysis, 3(3), 285-300. https://doi.org/10.1016/S1361-8415(99)80025-5.

14. Gonzales, A. V., Cinquin, P., Troccaz, J., Guerraz, A., Hennion, B., Pellissier, F., et al. (2001). TER: A system for robotic tele-echography. Medical image computing and computer-assisted intervention-MICCAI 2001: 4th International Conference Utrecht, The Netherlands, October 14-17, 2001 Proceedings (pp. 326-334). https://doi.org/10.1007/3-540-45468-3_39.

15. Kelly, K. M., Dean, J., Comulada, W. S., \& Lee, S.-J. (2010). Breast cancer detection using automated whole breast ultrasound and mammography in radiographically dense breasts. European Radiology, 20(3), 734-742. https://doi.org/10.1007/s0033 0-009-1588-y.

16. Chang, R.-F., \& Shen, Y.-W. (2013). 3D whole-breast ultrasonography (Citeseer).

17. Lee, C. Y., Truong, T. L., \& Li, P. C. (2017). Automated conformal ultrasound scanning for breast screening. Journal of Medical and Biological Engineering, 38(1), 116-128. https://doi. org/10.1007/s40846-017-0292-7.

18. Nguyen, C. V., Izadi, S., \& Lovell, D. modeling kinect sensor noise for improved 3D reconstruction and tracking. In Proceedings of the 2012 Second International Conference on 3D Imaging, Modeling, Processing, Visualization \& Transmission, 2012 (pp. 524-530). 2415802: IEEE Computer Society. https://doi. org/10.1109/3dimpvt.2012.84. 